\title{
Beyond age at first sex: Patterns of emerging sexual behavior in adolescence and young adulthood
}

\author{
Abigail A. Haydon, MPH, $\mathrm{PhD}^{1,2}$, Amy H. Herring, $\mathrm{ScD}^{2,3}$, Mitchell J. Prinstein, $\mathrm{PhD}^{4}$, and \\ Carolyn Tucker Halpern, $\mathbf{P h D}^{1,2}$ \\ ${ }^{1}$ Department of Maternal and Child Health, University of North Carolina at Chapel Hill, UNC \\ Gillings School of Global Public Health, Chapel Hill, NC \\ ${ }^{2}$ Carolina Population Center, University of North Carolina at Chapel Hill, Chapel Hill, NC \\ ${ }^{3}$ Department of Biostatistics, University of North Carolina at Chapel Hill, UNC Gillings School of \\ Global Public Health, Chapel Hill, NC \\ ${ }^{4}$ Department of Psychology, University of North Carolina at Chapel Hill, Chapel Hill, NC
}

\begin{abstract}
Purpose-Although the emergence of sexual expression during adolescence and early adulthood is nearly universal, little is known about patterns of initiation.

Methods-We used latent class analysis to group 12,194 respondents from Waves I and IV of the National Longitudinal Study of Adolescent Health (Add Health) into one of five classes based on variety, timing, spacing, and sequencing of oral-genital, anal, and vaginal sex. Multinomial logistic regression models, stratified by biological sex, examined associations between sociodemographic characteristics and class membership.
\end{abstract}

Results-Approximately half of respondents followed a pattern characterized predominately by initiation of vaginal sex first, average age of initiation of approximately 16 years, and spacing of one year or more between initiation of the first and second behaviors; almost one third initiated sexual activity slightly later but reported first experiences of oral-genital and vaginal sex within the same year. Classes characterized by postponement of sexual activity, initiation of only one type of behavior, or adolescent initiation of anal sex were substantially less common. Compared to White respondents, Black respondents were more likely to appear in classes characterized by initiation of vaginal sex first. Respondents from lower socioeconomic backgrounds were more likely to be in classes distinguished by early/atypical patterns of initiation.

Conclusions-A small number of typical and atypical patterns capture the emergence of sexual behavior during adolescence, but these patterns reveal complex associations among different elements of emerging sexuality that should be considered in future research.

(C) 2011 Society for Adolescent Medicine. Published by Elsevier Inc. All rights reserved.

Correspondence and requests for reprints should be sent to: Abigail Haydon, Carolina Population Center, University of North Carolina at Chapel Hill, CB\#8120, University Square, 123 West Franklin Street, Chapel Hill, NC 27516-2524, Phone: 650-776-6060, Fax: 919-966-6638, ahaydon@email.unc.edu.

Publisher's Disclaimer: This is a PDF file of an unedited manuscript that has been accepted for publication. As a service to our customers we are providing this early version of the manuscript. The manuscript will undergo copyediting, typesetting, and review of the resulting proof before it is published in its final citable form. Please note that during the production process errors may be discovered which could affect the content, and all legal disclaimers that apply to the journal pertain. 


\section{Keywords}

Oral sex; anal sex; latent class analysis; nationally representative; noncoital; sexuality development; adolescent sexual behavior; sociodemographic differences

The study of sexual development has traditionally emphasized the predictors and consequences of single sexual events over broad patterns of emerging sexual behavior - an approach at odds with both developmental systems perspectives on human behavior and the sexual/romantic experiences of adolescents and young adults. Addressing this imbalance is critical not only to the elaboration of theories of sexual development, but also to the design of effective interventions and curricula for promoting sexual health across the life course. The goal of this analysis is to describe patterns of initiation of oral-genital, anal and vaginal sex in a nationally representative sample, and the extent to which the prevalence of different patterns varies by sociodemographic characteristics.

\section{Patterns of early sexual behavior: Variety, timing, spacing, and sequencing}

The correlates of first vaginal sex are arguably the most widely studied aspect of adolescent sexuality [1]. Exclusive focus on vaginal sex, however, is inconsistent with cross-sectional research indicating substantial variety in adolescent sexual expression. Data from the 20062008 National Survey of Family Growth (NSFG) indicate that approximately 48\% of males and $45 \%$ of females have engaged in oral-genital sex with an opposite-sex partner, and approximately $11 \%$ of females and $10 \%$ of males ages $15-19$ have engaged in anal sex with an opposite sex partner [2].

While initiation of anal sex typically occurs well beyond adolescence (if at all), first experiences of oral-genital and vaginal sex appear to occur in close proximity [3]. In the first prospective study to examine the predictive relationship between initiation of oral-genital and vaginal sex, Halpern-Felsher et al. identified the modal behavioral pathway in a sample of California high school students as either initiation of oral-genital sex prior to vaginal sex or within the same six-month period [4]. In contrast, only 6\% of adolescents in the 2002 NSFG reported having had anal sex within six months of initiating vaginal sex [3]. These results suggest that certain sequences of sexual behaviors are statistically normative while others (e.g., anal sex prior to vaginal/oral-genital sex) are highly atypical.

Factors such as timing and sequencing of sexual behaviors vary by sociodemographic characteristics. In general, White adolescents are more likely than other racial/ethnic groups to report having engaged in oral-genital or anal sex $[3,5]$. Compared to White adolescents, Black adolescents initiate vaginal sex at earlier ages $[6,7]$ and are more likely to engage in vaginal sex prior to noncoital activities $[8,9]$. Early sexual behavior also differs by socioeconomic status, with some evidence that adolescents from higher socioeconomic backgrounds are more likely to engage in oral-genital or anal sex $[3,9]$.

Despite evidence of variation in the timing, sequencing, and spacing of coital and noncoital behaviors, we are aware of only one study that has examined these factors simultaneously [10]. The majority of respondents in this Dutch sample reported gradual and so-called "linear" sexual progressions (in which less intimate behaviors, such as kissing or heavy petting, proceeded more intimate behaviors, such as oral-genital or vaginal sex), while the remainder experienced a "nonlinear" and/or tightly spaced progression. The extent to which similar patterns are evident in US populations, however, is unclear. Moreover, the concept of linearity - which relies upon researchers' judgements of the relative intimacy of different behaviors - may be inappropriate for use in sociodemographically diverse populations, particularly given evidence that sexual norms may vary by race/ethnicity [11]. 
This line of research is important for several reasons. Unlike the narrow and decontextualized definition of sexuality encouraged by examining sexual behaviors in isolation, comprehensive description of broad patterns of early sexual behavior provides a basis for research conceptualizing sexuality as a normative developmental process. Basic description of behavioral patterns also facilitates contextual interpretation, builds the foundation for more advanced statistical modeling, and helps elaborate theories of sexual development that can inform prevention and intervention efforts [12]. Moreover, research in other behavioral domains - such as romantic relationship formation [13] - suggests that early sexual patterns may have important implications for promoting sexual health. From a practical standpoint, further delineation of sexual patterns - and sociodemographic differences in these patterns - is therefore critical to identifying interpersonal and behavioral contexts that may contribute to reproductive and sexual health disparities $[14,15]$.

\section{The present study}

Our primary aim was to describe typical and atypical patterns of early sexual development in a nationally representative sample of adolescents. We used latent class analysis (LCA) to identify these patterns, making no assumptions about the relative intimacy of different behaviors or the linearity of sexual patterns. Our secondary aim, after identifying classes, was to document sociodemographic differences in class membership.

\section{Methods \\ Data}

We used data from the National Longitudinal Study of Adolescent Health (Add Health), a nationally representative study of approximately 20,000 adolescents in the United States in grades 7-12 in 1994-1995. The Add Health design has been described in detail elsewhere [16]. To date, four waves of data collection have followed respondents from adolescence into adulthood. The present analysis used data from Wave I ( $N=20,745$; ages 11-21; response rate $=78.9 \%)$ and Wave IV $(\mathrm{N}=15,701$; ages $24-34$; response rate $=80.3 \%)$, and was restricted to respondents who appeared at both waves and had valid sampling weights $(\mathrm{N}=14,800)$. Respondents lacking data on lifetime history and ages of initiation of oralgenital, anal, or vaginal sex $(n=969)$ or sociodemographic characteristics $(n=243)$ were excluded. Since patterns of sexual development may differ substantially between sexual minority and sexual majority individuals $[17,18]$, we also excluded respondents who reported having had a same-sex romantic partner $(\mathrm{n}=1,526)$. These exclusion criteria yielded an analytic sample of 12,194 .

\section{Measures}

Sexual behavior-At Wave IV, respondents used computer self-interviewing technology (CASI) to report whether they had ever engaged in vaginal, anal, and oral-genital sex, based on the following questions. Vaginal sex: "Have you ever had vaginal intercourse? (Vaginal intercourse is when a man inserts his penis into a woman's vagina.)" Oral-genital sex: "Have you ever had oral sex? That is, has a partner ever put his/her mouth on your sexual organs or you put your mouth on his/her sex organs?" Anal sex: "Have you ever had anal intercourse? (By anal intercourse, we mean when a man inserts his penis into his partner's anus or butt hole.)" For each endorsed behavior, respondents indicated the age (in years) of initiation.

We used this information to generate five measures of sexual experience that serve as indicators of emergent sexual patterns in subsequent latent class analyses. We classified the first behavior initiated as vaginal sex, oral-genital sex, vaginal and oral-genital sex initiated within the same year, or anal sex (alone or within the same year as vaginal and/or oral- 
genital sex). Because ages of initiation were reported in whole years, we were unable to obtain more detailed sequencing information for respondents who reported initiating two or more behaviors at the same whole-digit age. Timing of first sexual experience was defined as the age at which the first sexual experience (among oral-genital, anal, and vaginal sex) occurred. Number/variety of behaviors indicated the number of different sexual behaviors (among the three measured) in which the respondent had ever engaged. Spacing between first and second behavior was defined as the age in years of the first sexual experience subtracted from the age of the second experience, and was converted to a six-category nominal variable (including a response category for "initiated only one type of behavior"). Lastly, we included an indicator of whether the respondent had initiated anal sex before age 18.

Sociodemographic characteristics-We created a four-category measure of parental educational attainment (less than high school; high school diploma or GED; some college or post-high school vocational education; or college graduate) using data from the Wave I parent interview. We selected the highest level attained in households with two resident parents. When parental reports were unavailable (approximately $15 \%$ of the total adolescent sample), we substituted the adolescent's report of their parents' educational attainment. We derived a combined measure of race/ethnicity (Hispanic, any race; non-Hispanic Black; nonHispanic White; and Other) from respondents' Wave I self-report. Wave IV chronological age was calculated by subtracting the date of birth from the Wave IV interview date. Biological sex was based on respondents' self-report.

\section{Analysis Plan}

Similar to cluster analysis, the goal of LCA is to group cases into similar classes based on responses to a given set of items $[19,20]$. We implemented latent class models in Latent Gold 4.5 (Statistical Innovations, Belmont, MA) and conducted analyses with 250 sets of random starting values, specifying 250 iterations for each set. There is no universally agreed-upon indicator for selecting the optimal number of classes in LCA [21]; we therefore relied on a number of different criteria. First, because our goal was to identify broad patterns of sexual development, we excluded class solutions that yielded latent class sizes of less than five percent of the full sample. We also examined the Bayesian Information Criterion (BIC) [22], a goodness-of-fit measure that penalizes the log-likelihood to show preference for more parsimonious models. Lastly, we considered interpretability and the extent to which classes were substantively different.*

Latent class membership is not directly observed. In order to model associations between sociodemographic factors and latent classes, we therefore assigned individuals to the class associxated with the highest individual-specific posterior probability. We then used multinomial logistic regression to assess whether sociodemographic variables predicted a greater likelihood of membership in certain classes compared to others. These models were implemented in Stata 11.0 (StataCorps, College Station, TX) and stratified by biological sex. All analyses used survey commands to adjust for Add Health's complex survey design and applied sampling weights to yield national population estimates.

\footnotetext{
* In preliminary LCAs stratified by sociodemographic characteristics (race/ethnicity, biological sex, parental educational attainment [college degree versus no college degree], and chronological age at Wave IV [24 to 28 versus 29 and above]), class structures were comparable across sociodemographic groups. We therefore present results based on analyses of the total sample.
} 


\section{Results}

\section{Sample characteristics}

Table 1 presents sociodemographic characteristics of our analytic sample. Overall, the average age of initiation was just over 16 years. Approximately equal percentages of respondents reported that they initiated vaginal sex first (39\%) or initiated vaginal and oralgenital sex within the same year (41\%). Six percent of respondents reported engaging in only one type of sexual behavior; the majority initiated a second behavior within two years or less. Almost $43 \%$ of respondents reported having engaged in all three types of behaviors (oral-genital, vaginal, and anal sex). Just under ten percent of respondents initiated anal sex before age 18 .

\section{Patterns of early sexual behavior}

We examined latent class solutions for three through seven classes, and selected the fiveclass solution on the basis of interpretability, meaningful distinctions among classes, BIC values, and classification error. This solution accounted for $80 \%$ of the variation in indicator variables. Table 2 describes the percentage distribution of each sexual behavior indicator, by latent class. Because any latent class analysis must balance model parsimony against homogeneity within classes, the behavioral profiles of respondents within each class are not identical. Instead, the latent class label reflects the modal behavioral profile for members of that class. Respondents who were likely to initiate vaginal sex first and then wait at least a year before initiating another behavior (typically oral-genital sex, since less than ten percent reported anal sex before age 18) comprised the largest class (Vaginal Initiators/Multiple Behaviors; 49\%). The second largest class (Dual Initiators; 32\%) consisted solely of respondents who initiated oral-genital and vaginal sex within the same year and did not have anal sex during adolescence. The third class (Vaginal Initiators/Single Behavior; 8\%) was distinguished by the fact that over three-quarters of its members had only engaged in one type of behavior (typically vaginal sex). The two smallest classes - Postponers (6\%) and Early/Atypical Initiators (6\%) - represented the most non-normative patterns. Postponers delayed sexual activity until almost 22 years of age, on average, but reported a relatively fast progression once initiation had occurred: $85 \%$ of respondents in this class initiated oralgenital and vaginal sex within the same year. In contrast, the Early/Atypical class was characterized by an early age of initiation ( 15 years, on average) in combination with initiation of two or more behaviors within the same year. Most significantly, all Early/ Atypical Initiators reported having had anal sex by age 18 .

\section{Bivariate associations between sociodemographic characteristics and sexual patterns}

The distribution of latent class membership differed significantly across categories of sociodemographic indicators among both males and females, with the exception of Wave IV age among males (Table 3). Regardless of race/ethnicity, the Vaginal Initiator/Multiple Behaviors class was generally the most heavily populated for both males and females. However, Black males were more heavily concentrated in this class (57\% of Black male respondents) than were White males (39\% of White male respondents). Among females, the Dual Initiators class contained a greater proportion of White females (30\%) than of any other race/ethnicity group, while the Vaginal Initiators/Single Behavior class contained a greater proportion of Black females (21\%) than any other group. The Vaginal Initiators/ Multiple Behaviors and Dual Initiators classes were the most common for both males and females across parental education categories, although substantial proportions of male respondents whose parents did not complete high school also appeared in the Vaginal Initiators/Single Behavior class (17\%) and Early/Atypical Initiators class (9\%). 


\section{Multivariate associations between sociodemographic characteristics and sexual patterns}

Table 4 presents results of multinomial logistic regressions modeling associations between sociodemographic characteristics and class membership, stratified by biological sex. Among both males and females, Black respondents were less likely than White respondents to appear in classes characterized by initiation of two or more behaviors within the same year and more likely to appear in classes in which vaginal sex was typically the first behavior initiated. Black males were more likely than their White counterparts to appear in the Vaginal Initiators/Single Behavior class (relative risk ratio [RRR]=1.8; 95\% CI: 1.2, 2.6) and less likely to appear in the Dual Initiators (RRR=0.4; 95\% CI: $0.3,0.5$ ), Postponers (RRR=0.3; 95\% CI: 0.2, 0.5), or Early/Atypical Initiators (RRR=0.2; 95\% CI: 0.1, 0.4) classes than in the Vaginal Initiators/Multiple Behaviors class. Similar to their male counterparts, Black females were also more likely to be in the Vaginal Initiators/Single Behavior class (RRR=3.4; 95\% CI: 2.5, 4.6) and less likely to be in the Dual Initiators (RRR $=0.3$; 95\% CI: 0.3, 0.4), Postponers (RRR $=0.4,95 \%$ CI: 0.2, 0.7), and Early/Atypical Initiators (RRR $=0.2 ; 95 \%$ CI: 0.1, 0.5) classes than in the Vaginal Initiators/Multiple Behaviors class.

In contrast, patterns of association between parent education and class membership differed somewhat by biological sex. Controlling for race/ethnicity and chronological age, female respondents whose parent(s) did not complete high school were more likely to appear in the Vaginal Initiators/Single Behaviors class than in the Vaginal Initiators/Multiple Behaviors class (RRR $=2.5 ; 95 \% \mathrm{CI}: 1.7,3.7)$ compared to respondents in a household where at least one parent had a college degree. Female respondents whose parent(s) completed high school but did not obtain a college degree were less likely to be classified as Postponers (high school diploma only: RRR=0.3; 95\% CI: 0.2 , 0.5 ; some college: $\mathrm{RRR}=0.5,95 \%$ CI: 0.3 , 0.8 ) than as Vaginal Initiators/Multiple Behaviors relative to their counterparts whose parent(s) obtained a college degree. Like females, males whose parent(s) did not complete high school were more likely than those with at least one college-educated parent to appear in the Vaginal Initiators/Single Behavior class than in the Vaginal Initiators/Multiple Behaviors class ( $R R R=2.9,95 \%$ CI: 1.8, 4.6). However, male respondents whose parents had no post-secondary education were also more likely to appear in the Early/Atypical Initiators class (less than high school: RRR=1.9; 95\% CI: 1.1, 3.0; high school diploma or GED: RRR $=1.9,95 \%$ CI: 1.2, 2.8) class than in the Vaginal Initiators/Multiple Behaviors class.

\section{Discussion}

This is the first study, to our knowledge, to examine emergent sexual patterns in a nationally representative US sample on the basis of variety, timing, sequencing, and spacing of first experiences of oral-genital, anal, and vaginal sex. Using latent class analysis, we identified five distinct behavioral profiles that explained over eighty percent of the variance in these indicators. Just under half of all respondents followed a pattern characterized predominately by initiation of vaginal sex prior to oral-genital or anal sex, average age of initiation of approximately 16 years, and a delay of one year or more between initiation of the first and second behaviors. A sizeable minority of adolescents - almost one third - initiated sexual activity slightly later but reported first experiences of oral-genital and vaginal sex within the same year. Patterns characterized by delayed onset of sexual activity, experience with only one type of sexual behavior, and early initiation of sexual activity combined with adolescent initiation of anal sex were substantially less common.

Notably, none of these classes was characterized by adolescent initiation of oral-genital sex followed by a substantial delay before initiation of vaginal sex. While the extent to which initiation of oral-genital sex accelerates or delays the onset of vaginal sex is not fully 
understood [23], both cross-sectional and prospective data suggest that "substitution" of oral-genital sex for vaginal sex is relatively rare. Instead, initiation of oral-genital sex appears to substantially increase the likelihood of initiating vaginal sex, at least in select samples [4]. Our results, based on a nationally representative sample, support these conclusions.

Although latent class structures were similar across sociodemographic groups, the likelihood of membership in each class was not. Most striking was our finding that non-Hispanic Black males and females were more likely to appear in classes in which vaginal sex was typically initiated first and were substantially less likely to appear in classes defined by adolescent initiation of anal sex, initiation of oral-genital and vaginal sex within the same year, or adolescent abstention from sexual activity, compared to their non-Hispanic White counterparts. Reasons for these differences are not fully understood, but likely reflect multiple mechanisms operating within and across various levels of influence. For example, Black adolescents may be less likely to delay vaginal sex because of patterns of disadvantage that reduce the opportunity costs of early sexual activity and encourage greater tolerance of adolescent sexual activity and childbearing [7, 24]. Cultural variation in the perceived acceptability and appeal of various sexual practices may also contribute to observed differences [25]. In their study of sexual practices in the United States, Lauman et al. reported that Blacks respondents were less likely to find either giving or receiving oral sex "very appealing" than Whites [26]. While they speculate that these differences are due to continued segregation of sexual networks that slows the diffusion of new sexual trends across groups, underlying causes of racial/ethnic differences in sexual patterns remain unclear.

Our results reveal complex associations among the behavioral characteristics that comprise broad sexual patterns. Respondents in the two classes largely characterized by initiation of vaginal sex first, for example, varied substantially in their ages of initiation and whether or not they went on to initiate noncoital behaviors. Similarly, age of initiation did not necessarily predict the extent to which sequences of initiation were spaced out over time: while the vast majority of both Dual Initiators and Postponers initiated oral-genital and vaginal sex within the same year, their average ages of initiation differed by over five years. Age of initiation was associated with variety of sexual experience, however. Early/Atypical Initiators reported the earliest ages of initiation and were also the only class defined by experience with all three behaviors. Taken together, these findings indicate that examining single sexual behaviors in isolation does not fully capture the complexity of emerging adolescent and young adult sexuality, and potentially overlooks aspects of early sexuality that may have important implications for subsequent health and wellbeing.

\section{Limitations}

Strengths of this analysis include use of a nationally representative and

sociodemographically diverse sample, inclusion of both coital and noncoital behaviors, and attention to other elements of sexual development beyond just the timing of the first coital experience. However, our reliance on retrospective self-reported data entails several limitations. Particularly for older respondents who initiated sexual activity at an early age, recall of ages of initiation may be inaccurate.* Moreover, because respondents reported ages of initiation in whole years, we were unable to determine the temporal ordering of behaviors

\footnotetext{
*In supplemental analyses, we explored the consistency of these reports by comparing respondents' reports of the age of first vaginal sex at Wave III (when respondents were between the ages of 18 and 26) and Wave IV (when respondents were 26 to 32). While a substantial proportion of respondents reported inconsistent ages, the vast majority of inconsistencies were relatively small in magnitude: approximately $84 \%$ of respondents either reported the same age at Wave III and Wave IV or reported an absolute difference of only one year.
} 
that occurred at the same age. Data limitations also prevented us from considering a broader range of sexual behaviors (e.g., masturbation, kissing, etc.), the interpersonal context in which behaviors occurred, and the voluntariness of first sexual experiences.

\section{Conclusions}

Prior research on adolescent sexuality has revealed little about how partnered sexual activity unfolds. By simultaneously examining timing, sequencing, spacing, and variety of behaviors, we were able to more completely describe patterns of emerging sexuality among US adolescents. This work contributes to growing interest in the full repertoire of adolescent sexual and romantic experiences, the processes by which these behaviors interact with biological, psychological, and social factors to contribute to adult health and wellbeing, and the role of emerging sexuality in the context of other developmental processes [27, 28]. Research on whether and how early sexual patterns contribute to adult sexual and reproductive health should be a priority, as both researchers and policymakers seek to promote healthy sexuality across the life course.

\section{Acknowledgments}

This research uses data from Add Health, a program project directed by Kathleen Mullan Harris and designed by J. Richard Udry, Peter S. Bearman, and Kathleen Mullan Harris at the University of North Carolina at Chapel Hill, and funded by grant P01-HD31921 from the Eunice Kennedy Shriver National Institute of Child Health and Human Development, with cooperative funding from 23 other federal agencies and foundations. Special acknowledgment is due Ronald R. Rindfuss and Barbara Entwisle for assistance in the original design. Information on how to obtain the Add Health data files is available on the Add Health website (http://www.cpc.unc.edu/addhealth). No direct support was received from grant P01-HD31921 for this analysis. Effort by Ms. Haydon and Ms. Halpern was supported by the Eunice Kennedy Shriver National Institute of Child Health and Human Development (grant R01HD57046, CT Halpern, Principal Investigator).

\section{References}

1. Zimmer-Gembeck MJ, Helfand M. Ten years of longitudinal research on US adolescent sexual behavior: Developmental correlates of sexual intercourse, and the importance of age, gender and ethnic background. Dev Rev. 2008; 28:153-224.

2. Chandra, A.; Mosher, WD.; Copen, C., et al. Sexual behavior, sexual attraction, and sexual identity in the United States: Data from the 2006-2008 National Survey of Family Growth. Hyattsville, MD: National Center for Health Statistics; 2011.

3. Lindberg LD, Jones R, Santelli JS. Noncoital sexual activities among adolescents. J Adolesc Health. 2008; 43:231-238. [PubMed: 18710677]

4. Song AV, Halpern-Felsher BL. Predictive relationship between adolescent oral and vaginal sex: Results from a prospective, longitudinal study. Arch Pediatr Adolesc Med. 2001; 165:243-249. [PubMed: 21041589]

5. Leichliter JS, Chandra A, Liddon N, et al. Prevalence and correlates of heterosexual anal and oral sex in adolescents and adults in the United States. J Infect Dis. 2007; 196:1852-1859. [PubMed: 18190267]

6. Cavazos-Rehg PA, Krauss MJ, Spitznagel EL, et al. Age of sexual debut among US adolescents. Contraception. 2009; 80:158-162. [PubMed: 19631791]

7. Furstenberg FF, Morgan SP, Moore KA, et al. Race differences in the timing of adolescent intercourse. Am Sociol Rev. 1987; 52:511-518.

8. Ompad DC, Strathdee SA, Celentano DD, et al. Predictors of early initiation of vaginal and oral sex among urban young adults in Baltimore, Maryland. Arch Sex Behav. 2006; 35:53-65. [PubMed: 16502153]

9. Brewster KL, Tillman KH. Who’s doing it? Patterns and predictors of youths' oral sexual experiences. J Adolesc Health. 2008; 42:73-80. [PubMed: 18155033] 
10. de Graaf H, Vanwesenbeeck I, Meijer S, et al. Sexual trajectories during adolescence: Relation to demographic characteristics and sexual risk. Arch Sex Behav. 2009; 38:276-282. [PubMed: 18165892]

11. Smith EA, Udry JR. Coital and non-coital sexual behaviors of white and black adolescents. Am J Public Health. 1985; 75:1200-1203. [PubMed: 4037163]

12. Shanahan MJ, Erickson LD, Bauer DJ. One hundred years of knowing: The changing science of adolescence, 1904 and 2004. J Res Adolesc. 2005; 15:383-394.

13. Meier A, Allen G. Romantic relationships from adolescence to young adulthood: Evidence from the National Longitudinal Study of Adolescent Health. Sociol Q. 2009; 50:308-335.

14. Halpern CT, Hallfors D, Bauer DJ, et al. Implications of racial and gender differences in patterns of adolescent risk behavior for HIV and other sexually transmitted diseases. Perspect Sex Repro Health. 2004; 36:239-247.

15. Hallfors DD, Iritani BJ, Miller WC, et al. Sexual and drug behavior patterns and HIV and STD racial disparities: The need for new directions. Am J Public Health. 2007; 97:125-132. [PubMed: 17138921]

16. Harris, KM.; Halpern, CT.; Entzel, P., et al. [Accessed January 16, 2011] The National Longitudinal Study of Adolescent Health: Research Design. Available at: http://www.cpc.unc.edu/projects/addhealth/design

17. Diamond LM. Female bisexuality from adolescence to adulthood: Results from a 10-year longitudinal study. Dev Psychol. 2008; 44:5-14. [PubMed: 18194000]

18. Savin-Williams RC, Ream GL. Prevalence and stability of sexual orientation components during adolescence and young adulthood. Arch Sex Behav. 2007; 36:385-394. [PubMed: 17195103]

19. Goodman LA. Exploratory latent structure analysis using both identifiable and unidentifiable models. Biometrika Trust. 1974; 61:215-231.

20. McCutcheon, AC. Latent Class Analysis. Beverly Hills, California: Sage Publications; 1987.

21. Nylund KL, Asparoutiov T, Muthen BO. Deciding on the number of classes in latent class analysis and growth mixture modeling: A Monte Carlo simulation study. Struct Equ Modeling. 2007; 14:535-569.

22. Schwarz G. Estimating dimension of a model. Ann Stat. 1978; 6:461-464.

23. Halpern-Felsher B. Oral sexual behavior: Harm reduction or gateway behavior? J Adolesc Health. 2008; 43:207-208. [PubMed: 18710673]

24. East PL. Racial and ethnic differences in girls' sexual, marital, and birth expectations. Journal of Marriage and the Family. 1998; 60:150-162.

25. Halperin DT. Heterosexual anal intercourse: Prevalence, cultural factors, and HIV infection and other health risks, Part I. AIDS Patient Care STDs. 1999; 13:717-730. [PubMed: 10743535]

26. Laumann, EO.; Gagnon, JH.; Michael, RT., et al. The Social Construction Sexuality: Sexual Practices in the United States. Chicago, Illinois: University of Chicago Press; 1994.

27. Graber, JA.; Brooks-Gunn, J.; Galen, BR. Betwixt and Between: Sexuality in the Context of Adolescent Transitions. In: Jessor, R., editor. New Perspectives on Adolescent Risk Behavior. Cambridge: Cambridge University Press; 1998.

28. Halpern CT. Reframing research on adolescent sexuality: healthy sexual development as part of the life course. Perspect Sex Reprod Health. 2010; 42:6-7. [PubMed: 20415876] 


\section{Implications and Contributions}

Examining the timing, sequencing, spacing, and variety of early sexual behaviors provides a more complete picture of adolescent and young adult sexual behavior, and highlights the importance of considering the multiple interactive factors that contribute to sexuality development. 


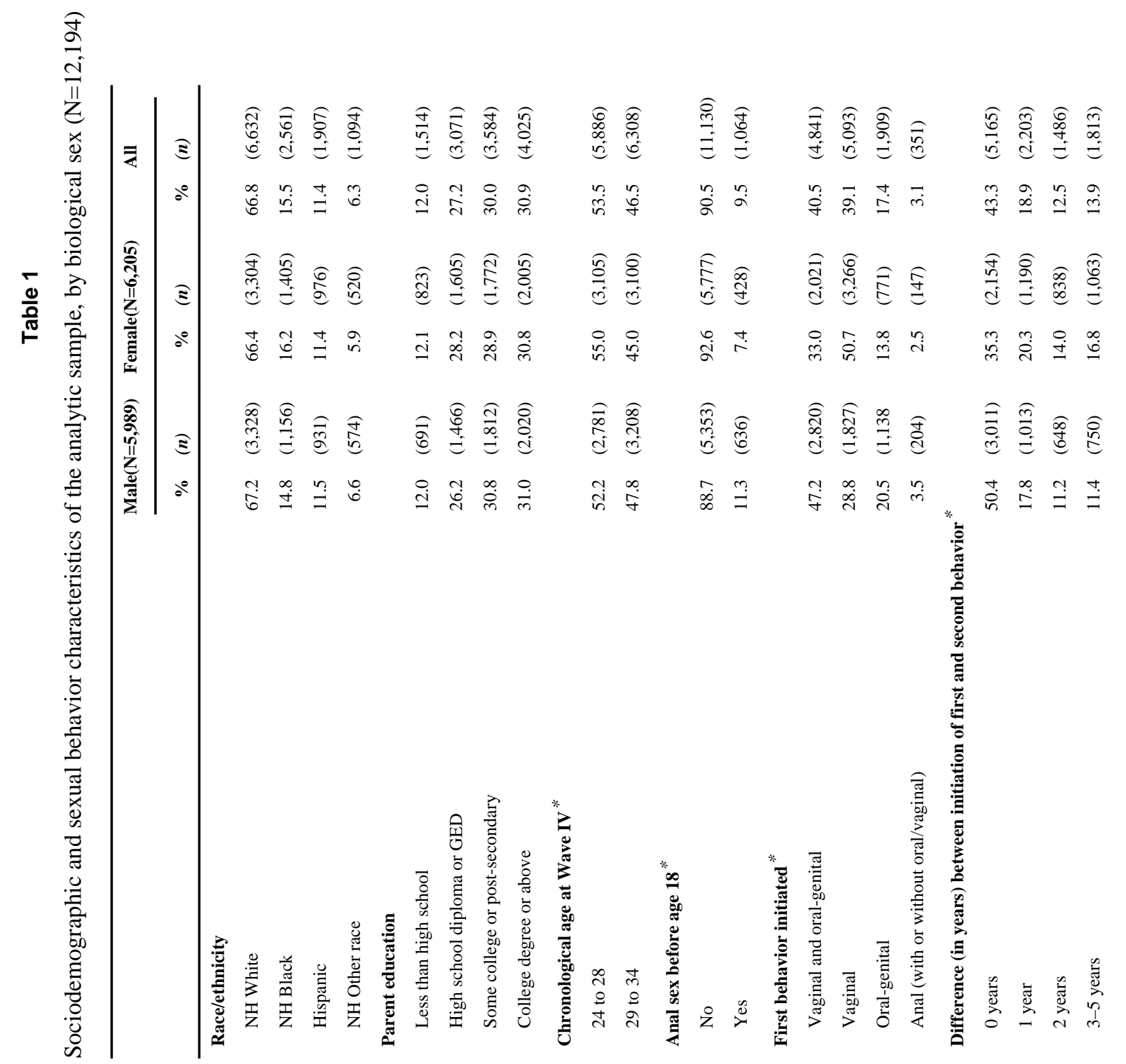




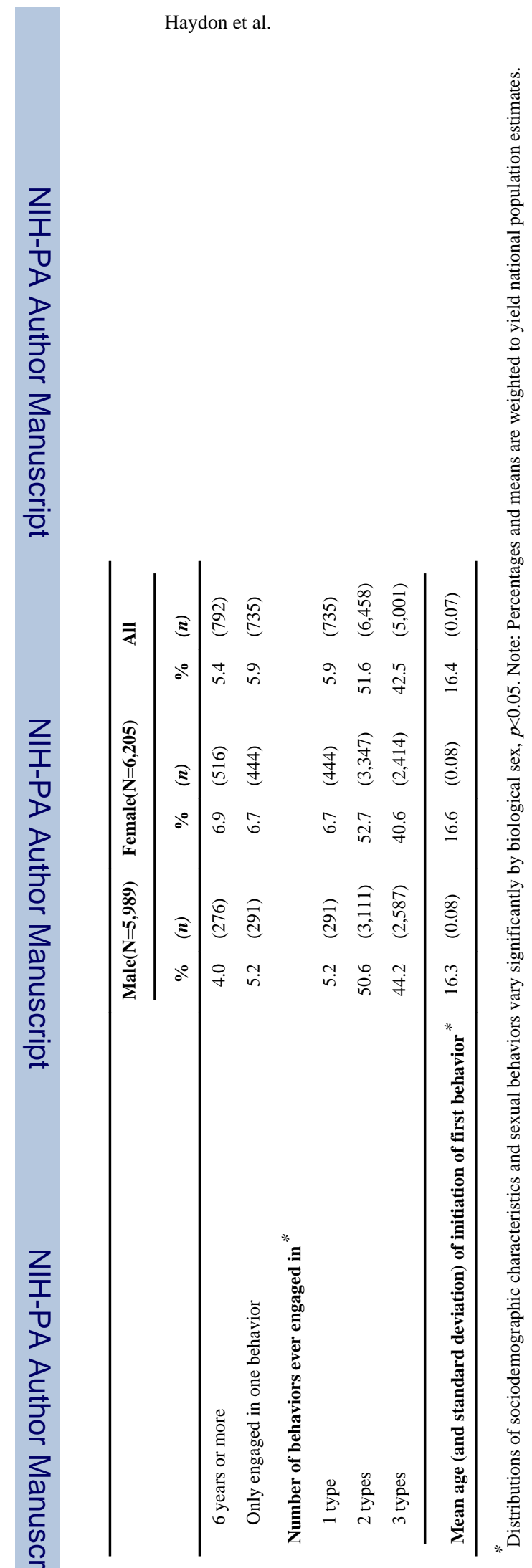

$J$ Adolesc Health. Author manuscript; available in PMC 2013 May 01. 


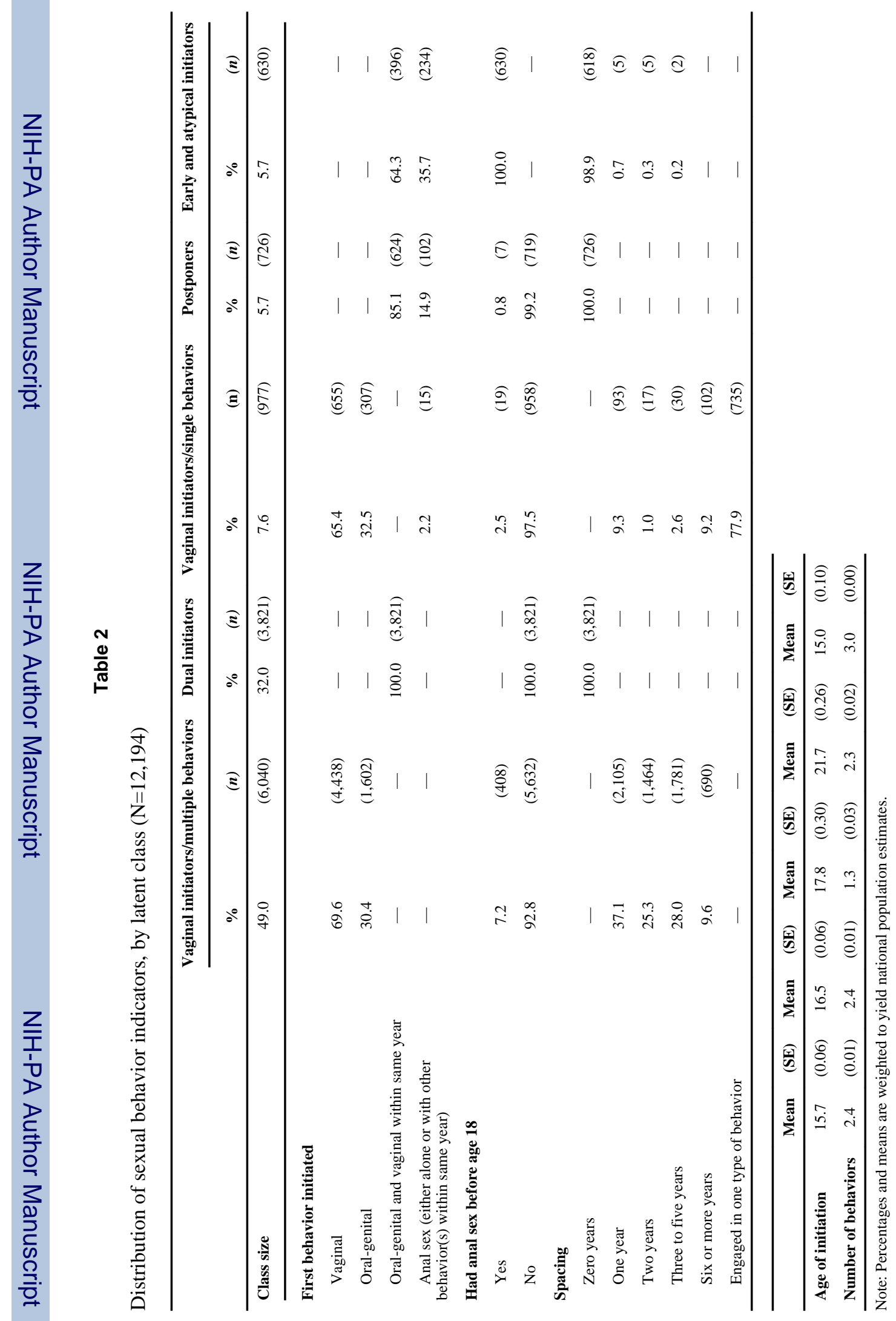




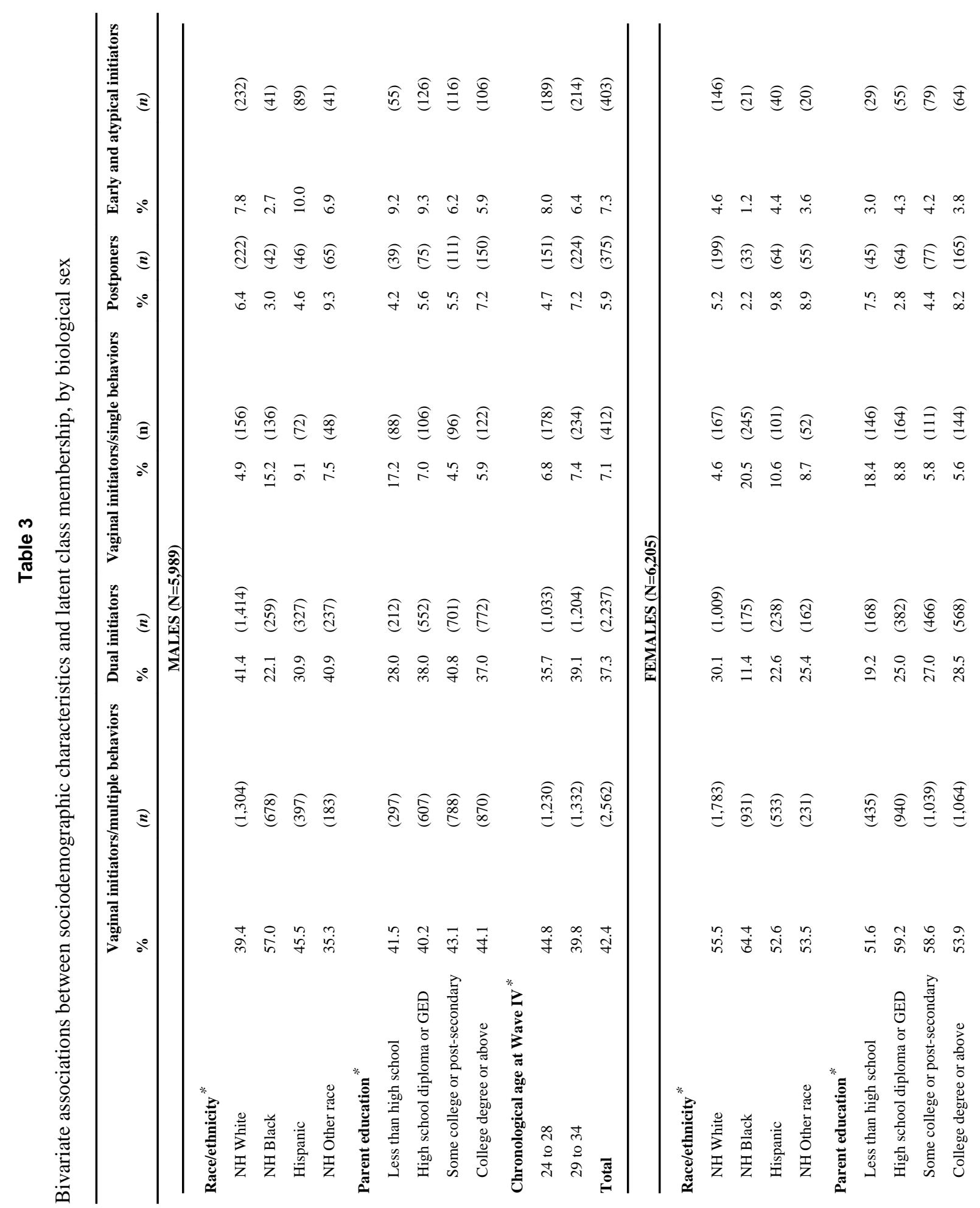


Haydon et al.

Page 15

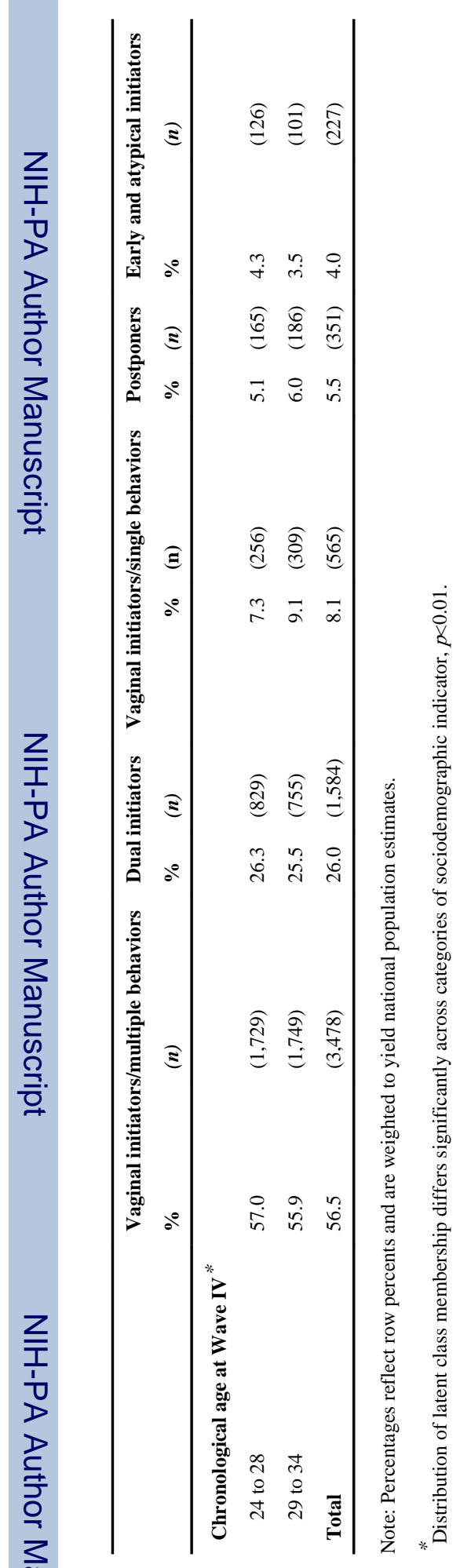

号

J Adolesc Health. Author manuscript; available in PMC 2013 May 01. 


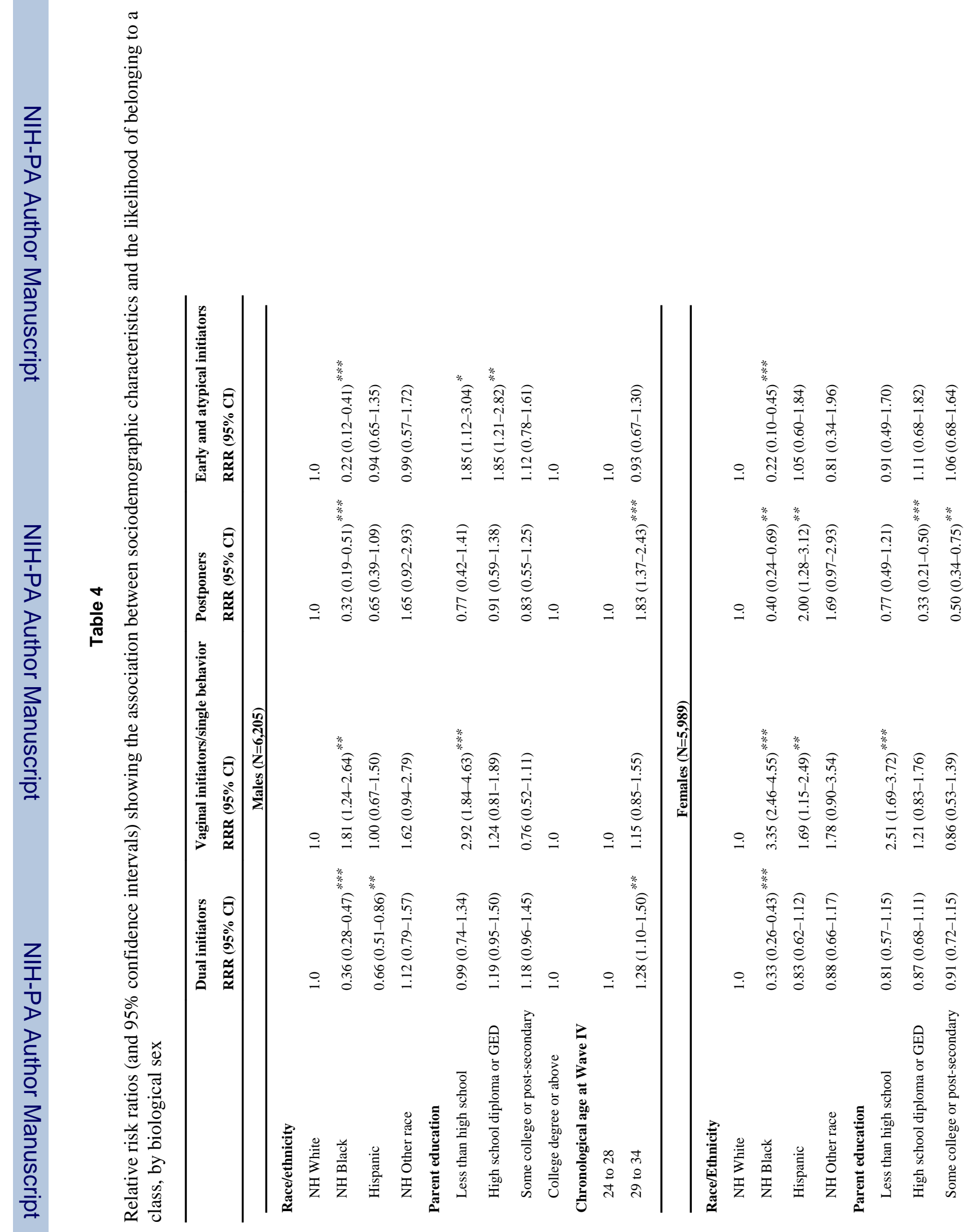




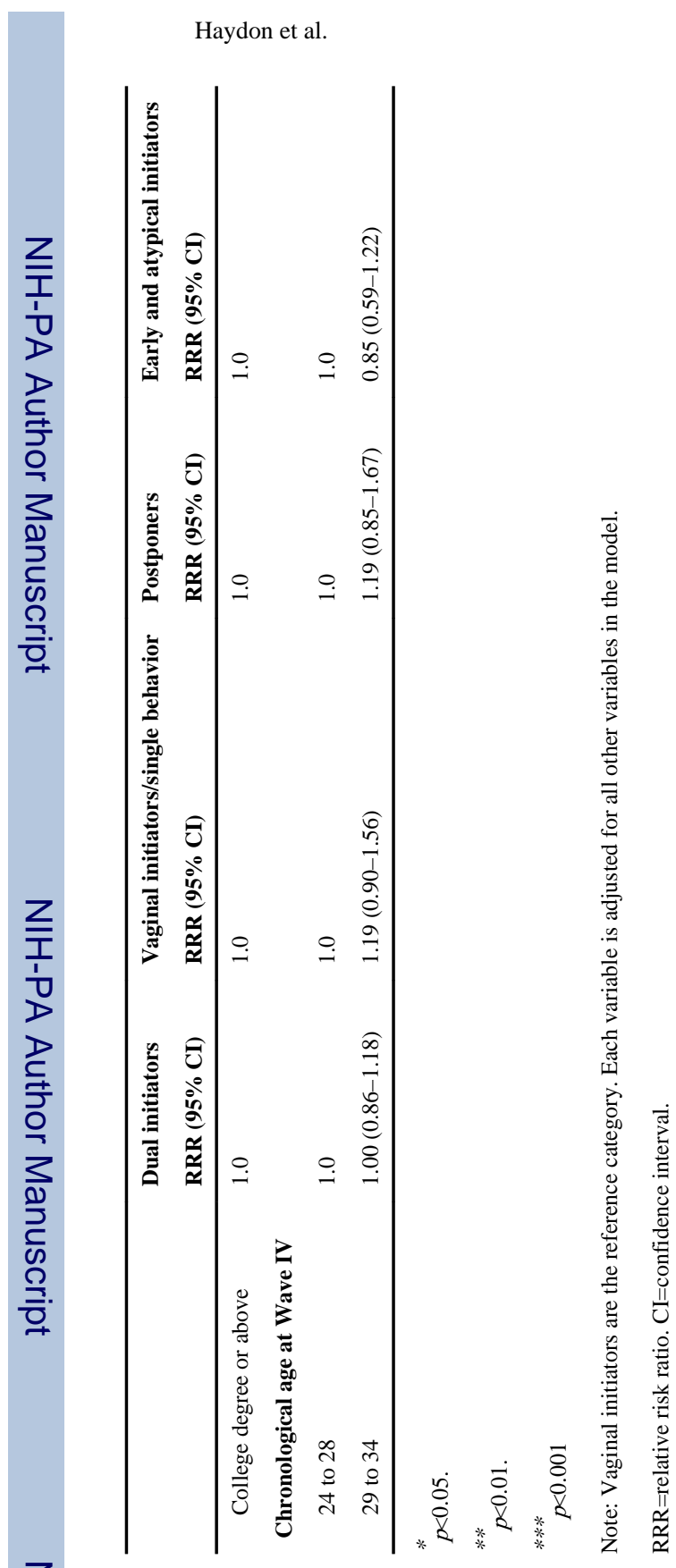

Page 17

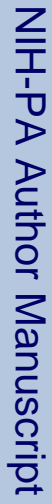

J Adolesc Health. Author manuscript; available in PMC 2013 May 01. 